\title{
Strongly coupled plasmas obey the fluctuation theorem for entropy production
}

\author{
Chun-Shang Wong ${ }^{\star}$, J. Goree, Zach Haralson and Bin Liu
}

\begin{abstract}
Fluctuation theorems ${ }^{1-4}$ describe nonequilibrium stochastic behaviour in small systems. Whilst experiments have shown that fluctuation theorems are obeyed by single particles in liquids ${ }^{5}$ and several other physical systems ${ }^{6-10}$, it has not been shown if that is the case in strongly coupled plasmas. Plasmas are said to be strongly coupled when interparticle potential energies are large compared to kinetic energies. Charged particles in such plasmas can behave collectively like liquids ${ }^{11,12}$, but with essential differences, such as long-range collisions ${ }^{13}$. It remains unexplored whether, despite these differences, the stochastic behaviour of strongly coupled plasmas will obey fluctuation theorems. Here we demonstrate experimentally that a strongly coupled dusty plasma obeys the fluctuation theorem of Evans, Cohen, and Morriss (ECM) ${ }^{14}$, which was developed for a simple liquid in a nonequilibrium steady state. This fluctuation theorem describes the entropy production arising from collisions in a steady laminar shear flow.
\end{abstract}

A dusty plasma ${ }^{15-17}$ is a four-component mixture of microspheres, electrons, positive ions, and a rarefied neutral gas, which all share a volume $e^{18}$. The microspheres, which are the heaviest of these components, develop large charges ${ }^{19}$ so that they become strongly coupled $^{20}$. The lighter charged components of the dusty plasma (electrons and positive ions) are weakly coupled. Dusty plasmas have much in common with other strongly coupled plasmas, such as ultracold neutral plasmas ${ }^{21}$, and warm dense matter ${ }^{22}$ as well.

The collection of microspheres can undergo a liquid-like flow when external forces are applied by laser beams ${ }^{23-27}$. In this way, the microspheres can be driven into a shear flow-that is, a flow with a transverse gradient in the flow velocity. In the shear flow, entropy production results from collisions between microspheres.

Many fluctuation theorems centre on the rate of entropy production in nonequilibrium systems below the thermodynamic limit. Fluctuation theorems (not to be confused with the similarly named fluctuation-dissipation theorem) all spawned from the ECM fluctuation theorem; this original fluctuation theorem was developed especially for a steady-state laminar shear flow. In a shear flow, entropy production is generated by viscous heating. This viscous heating is always positive in the thermodynamic limit, but it can fluctuate briefly to negative values for a subsystem within the fluid, containing a small number of molecules. These fluctuations, with negative heating and therefore negative entropy production, have been described as violations of the second law of thermodynamics ${ }^{14}$.

The ECM fluctuation theorem compares these negative-entropyproduction fluctuations to the more common positive fluctuations. In particular, the probabilities of these two kinds of fluctuations are predicted to have a ratio obeying ${ }^{14,28}$

$$
\ln \left[\frac{p\left(\sigma_{\tau}=-C\right)}{p\left(\sigma_{\tau}=C\right)}\right]=-C \tau \quad \text { as } \tau \rightarrow \infty
$$

We will later summarize equation (1), which is the historically important ECM fluctuation theorem, as left-hand side equals righthand side (LHS $=$ RHS), as $\tau \rightarrow \infty$. In equation $(1), \sigma_{\tau}$ is the entropy production rate averaged over an observation time $\tau$, and $p\left(\sigma_{\tau}=-C\right)$ is the probability that $\sigma_{\tau}$ has a specified negative value $-C$.

In addition to our main purpose of demonstrating that strongly coupled plasmas can obey a fluctuation theorem, our experiment serves also to reveal the robustness of the ECM fluctuation theorem. We do this by showing that beyond the original intended system of a simple liquid in a shear flow, ECM also works for a system with additional complications. These complications, for a dusty plasma, include long-range potentials and an open system for energy flow. The long-range screened Coulomb potentials for the microspheres are known to lead to distinctive behaviour such as a minimum in viscosity with respect to temperature ${ }^{29}$. The open system character of the collection of microspheres arises from flows of energy to and from the microspheres. Laser heating provides an external energy input, as does a powered electrode that sustains electric fields. These fields, which are modified by the electrons and ions, drive an ion flow that can give some of its energy to the microspheres. The combination of these energy inputs is balanced by an energy loss to the neutral gas due to friction. We will find that, despite all these complications, ECM is so robust that it accurately describes the fluctuations in entropy production in our collection of microspheres.

Our dusty plasma consisted of polymer microspheres suspended in a weakly ionized argon gas. The approximately $10^{4}$ microspheres were electrically confined to a single two-dimensional layer within the three-dimensional volume filled by the other dusty plasma components. The microspheres, of $8.69 \mu \mathrm{m}$ diameter, accumulated a large negative charge $Q=-15,000 e$, where $e$ is the elementary charge. Their mutual repulsion caused the microspheres to be spaced with a Wigner-Seitz radius $a=0.33 \mathrm{~mm}$. The microspheres collectively had an irregular liquid-like arrangement, due a kinetic temperature that was elevated using two laser beams ${ }^{24}$. In addition to this laser heating, a separate pair of laser beams was used to drive the collection of microspheres into a laminar shear flow.

The microsphere motion resulting from laser manipulation in our experiment is shown in Fig. 1. Representative microsphere trajectories in Fig. 1a show that the flow was straight and laminar, and that individual microspheres not only participated in the overall flow but also exhibited their own random thermal motion. The hydrodynamic profiles in Fig. 1b,c confirm that the kinetic temperature $T$ is uniform, and the flow velocity profile is linear within $9.0<y<11.5 \mathrm{~mm}$, which is the central region that we analyse.

We measured the local entropy production rate associated with viscous heating among the collection of microspheres. The instantaneous rate $\mathrm{e}^{14}, \sigma(t)=-P_{x y}(t) \gamma / k_{\mathrm{B}} T$, is calculated from the positions and velocities of microspheres and the binary 
a

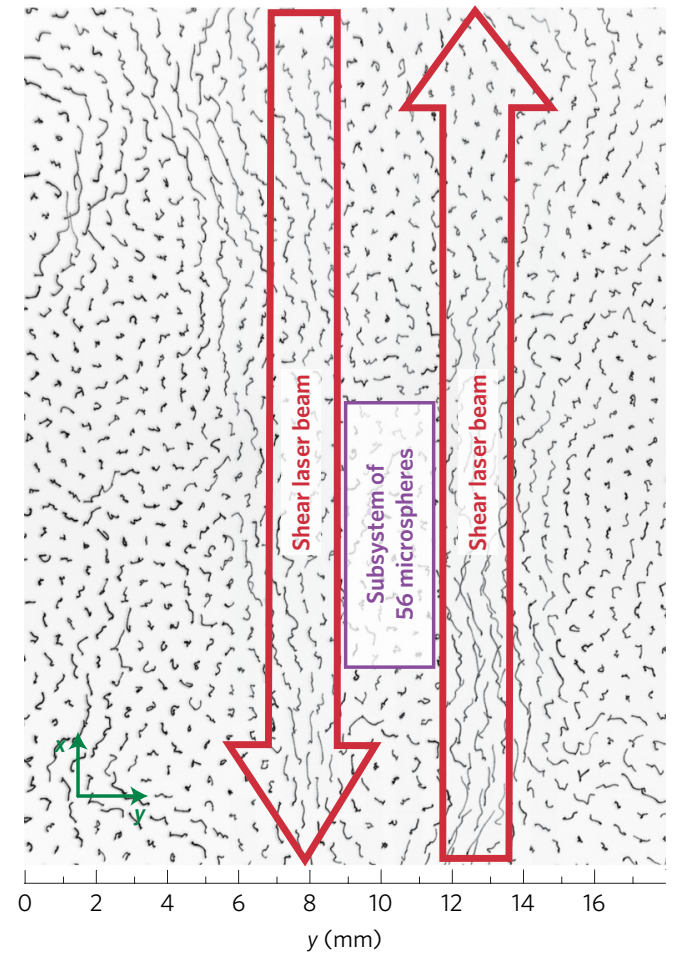

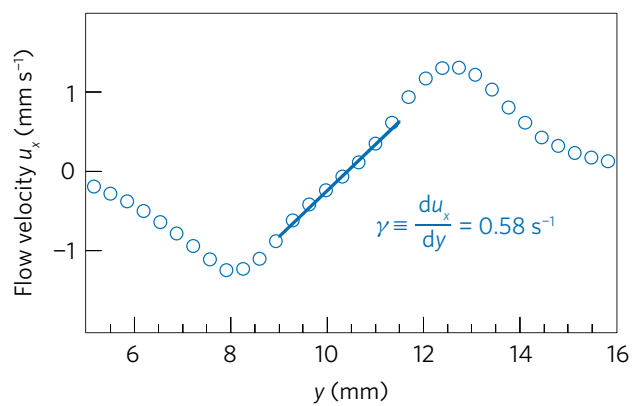

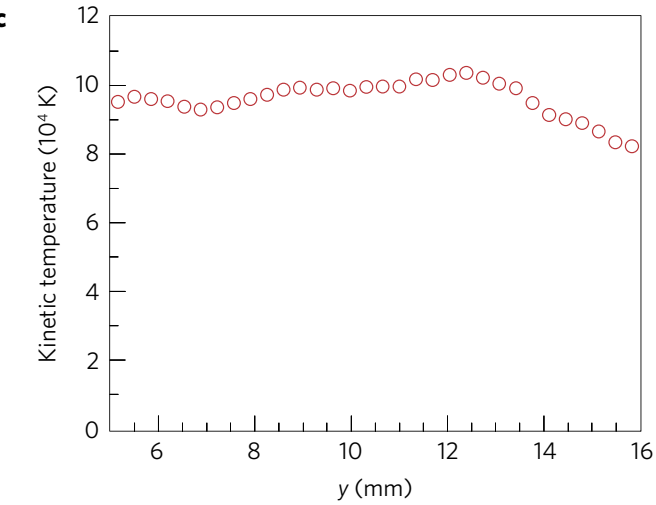

Figure 1 | Microsphere trajectories and hydrodynamic profiles in the dusty plasma experiment. Thousands of mutually repulsive charged microspheres

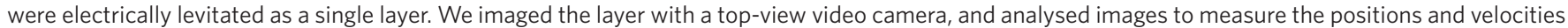
of individual microspheres ${ }^{30}$. a, Tracks of individual microspheres in the camera's field of view are shown over a duration of $0.3 \mathrm{~s}$, revealing a combination of random thermal motion and directed flow. The thermal and flow velocity were of the same order $\left(1 \mathrm{~mm} \mathrm{~s}^{-1}\right)$. The collective flow of the microspheres, which was laminar, was driven by the radiation pressure force of oppositely directed shear laser beams (as shown). Simultaneously, heating was applied separately ${ }^{24}$ over a much larger area by two other laser beams (not shown here) to sustain liquid-like conditions in the microsphere layer. We direct our attention to a specific subsystem, shown as a rectangle containing $N=56$ microspheres. $\mathbf{b}$, The flow pattern of the microspheres is a shear flow, with a linear gradient in the central region. This gradient $\gamma$ appears in the Navier-Stokes energy equation term $\mu \gamma^{2}$, where $\mu$ is the viscosity and $\gamma=\partial u_{x} / \partial y$. A non-zero value of $\gamma$ gives rise to viscous heating, which corresponds to entropy production. We obtained the hydrodynamic flow velocity profile as an average of the microsphere velocities. c, The hydrodynamic kinetic temperature profile is nearly uniform in the central region. This temperature was obtained from the mean-square microsphere velocities after subtracting the local flow velocity.

microsphere-microsphere interaction forces. Other forces, whether from an external source or from other components of the dusty plasma, do not enter into the expression above and therefore do not affect the entropy production that we measure, as explained in the Supplementary Information. Here, $P_{x y}(t)$ is the instantaneous shear stress and $\gamma$ is the shear rate (that is, the transverse gradient in the flow velocity).

In our experiment, video microscopy and tracking of the individual microspheres provided the necessary inputs to compute the shear stress ${ }^{26}, P_{x y}(t)$, and in turn, $\sigma(t)$ in a shear flow. The resulting time series $\sigma(t)$, for a subsystem of $N=56$ microspheres, is the basis of all our remaining analysis; a portion of this time series is shown in Fig. 2a.

To assess whether our liquid-like strongly coupled plasma obeys ECM, we need distributions of the time-averaged entropy production rate $\sigma_{\tau}$. We prepared these distributions as histograms, like Fig. 2b,c. This was done by averaging the $\sigma(t)$ time series within segments of duration $\tau$, yielding $\sigma_{\tau}$. Then, histograms were made by counting the events when $\sigma_{\tau}$ fell within a specified bin. Of particular interest are the negative fluctuations of $\sigma_{\tau}$, which can be seen in the shaded regions of Fig. 2b,c.

Counts in the experimentally obtained histograms, like those in Fig. 2b,c, were used as the probabilities $p$ in the LHS of the ECM fluctuation theorem, equation (1). We first chose a value of $\tau$ and selected two oppositely valued histogram bins, $\sigma_{\tau}= \pm C$. Then, we calculated the ratio of the counts in the bins for $-C$ and $+C$, allowing us to obtain the LHS using equation (1). The RHS was also calculated from $C$ and $\tau$. Finally, we compare the LHS to the RHS of equation (1) to test whether our strongly coupled plasma obeys the ECM fluctuation theorem. These results are presented in Fig. 3a,b, for a long $\tau$ and a short $\tau$, respectively.

Our chief result is that the ECM fluctuation theorem is obeyed by the strongly coupled component of our dusty plasma-the microspheres. This is demonstrated experimentally in Fig. 3a, where the LHS and RHS pairs of data points agree within error bars, for equation (1). We note that this agreement did not require adjusting any free parameter.

This agreement also demonstrates that, as a description of a shear flow, ECM is not limited to its original scope of simple liquids. Our experiment shows that ECM also accurately describes a shear flow for one component of a more complicated system.

Another aspect of the ECM fluctuation theorem that we can explore is its asymptotic behaviour. According to equation (1), the LHS and RHS must converge together for increasing $\tau$. This gradual tendency can be seen in our experimental data by comparing Fig. 3a and b. In Fig. 3a, for a long $\tau$, the LHS and RHS have converged together, but in Fig. $3 \mathrm{~b}$, for a short $\tau$, they have not converged.

The timescale for this convergence is a quantity that should be useful, in general, for gaining an understanding of a physical system. However, the fluctuation theorem itself is silent upon the matter of this timescale. Moreover, the literature says little about methods of measuring convergence time.

We have devised a prescription for precisely obtaining a characteristic time for convergence, $t_{c}$. In Fig. 4 , we use a curve $D(\tau)$, 
a

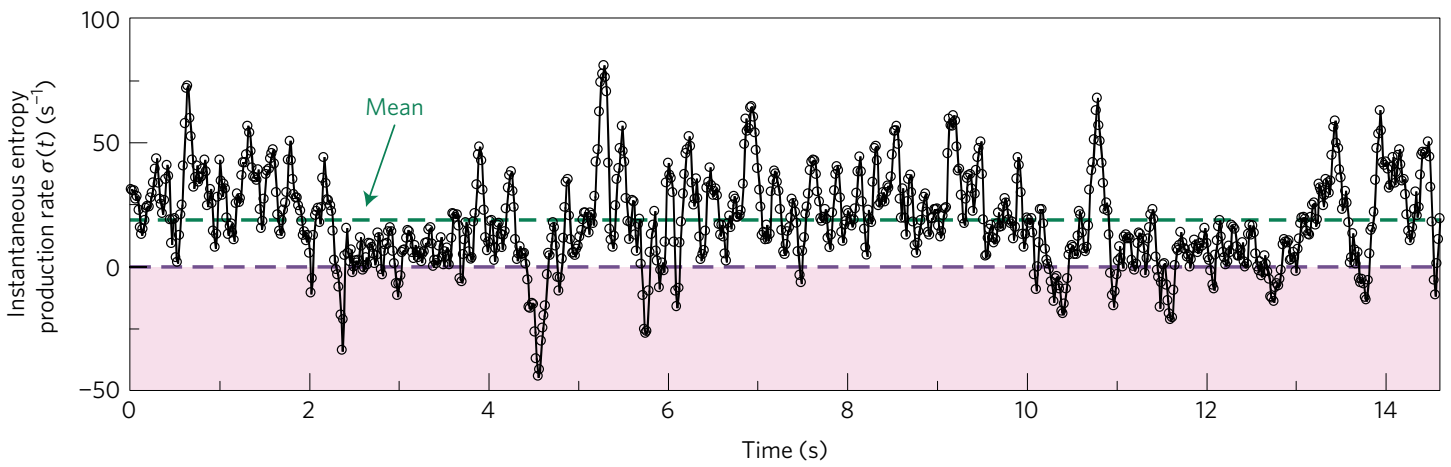

b

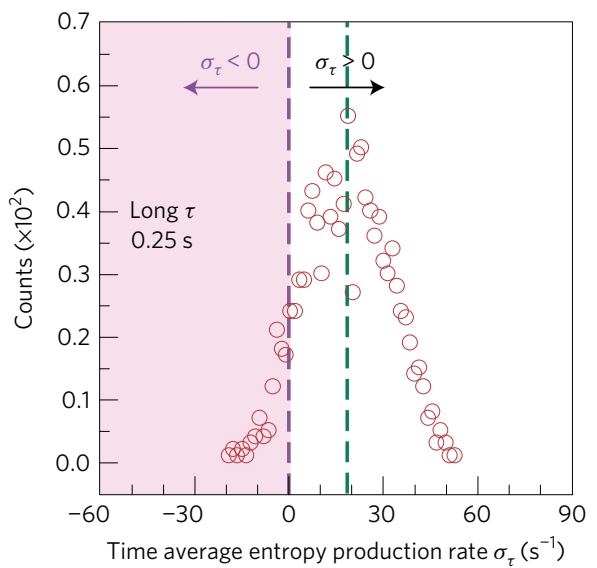

c

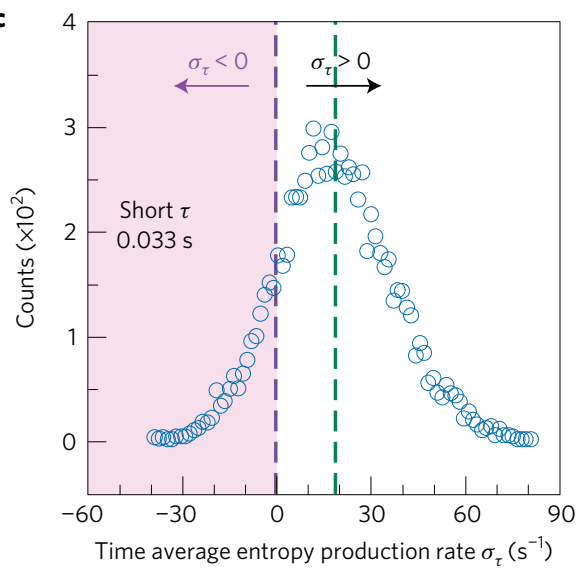

Figure 2 | Time series and histograms of entropy production rate. Using our particle-level experimental data, we obtain the rate of entropy production for a subsystem of $N=56$ microspheres. We report both the instantaneous rate $\sigma(t)$ and an average rate $\sigma_{\tau}=\left\langle-P_{x y}(t) \gamma / k_{\mathrm{B}} T\right\rangle_{\tau}$ for various observation times $\tau$, where $P_{x y}(t)$ is the shear stress and $\langle\ldots\rangle_{\tau}$ is a time average. a, Time series for the first $20 \%$ of the data set for the instantaneous entropy production rate. Second law violations are seen when $\sigma(t)$ fluctuates to negative values in the shaded region. Whilst $\sigma(t)$ does fluctuate to these negative values, it is more often positive, with a positive average value as required by the second law. $\mathbf{b}, \mathbf{c}$. Histograms of the time-averaged rate $\sigma_{\tau}$ also exhibit negative fluctuations, but less so for a long observation time $\tau$ (b) than for a short $\tau$ (c). Such histograms are used as the inputs for the LHS of equation (1).
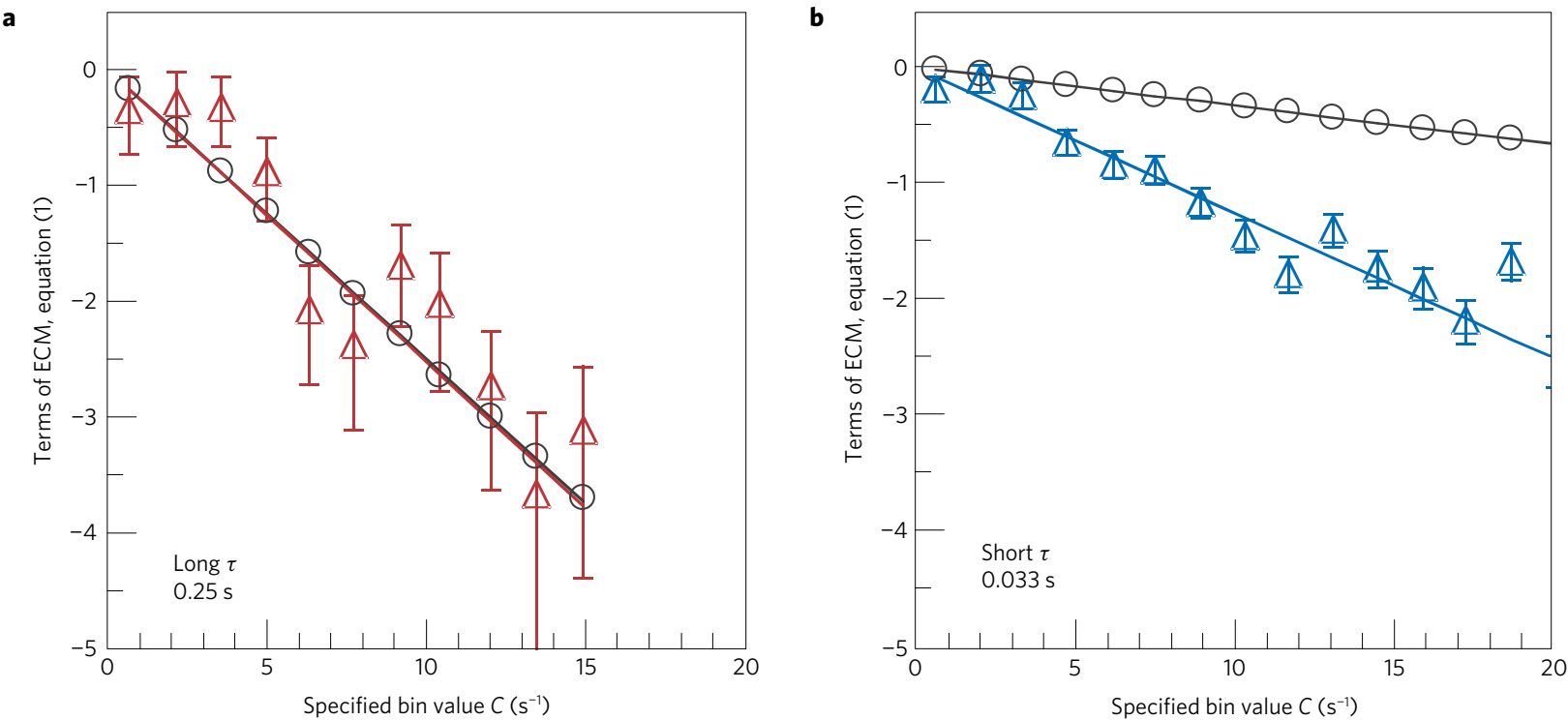

Figure 3 | Demonstration that a strongly coupled plasma obeys the ECM fluctuation theorem. We compare the two dimensionless terms of equation (1), the left-hand side (LHS, triangles) and right-hand side (RHS, circles), by examining a pair of data points for a given value of $C$. Most pairs of data points agree within error bars for a long $\tau$ (in $\mathbf{a}$ ), but not for a short $\tau$ (in $\mathbf{b}$ ); this result demonstrates that our data obey the ECM fluctuation theorem, including the asymptotic property of equation (1) that LHS $=$ RHS only as $\tau \rightarrow \infty$. The LHS data points in $\mathbf{a}, \mathbf{b}$ were obtained using histograms of $\sigma_{\tau}$, Fig. $2 b, \mathrm{c}$. Error bars, which represent one-standard-deviation uncertainties, were found from counting statistics for these histograms. Straight lines are drawn through the data points to guide the eye. 


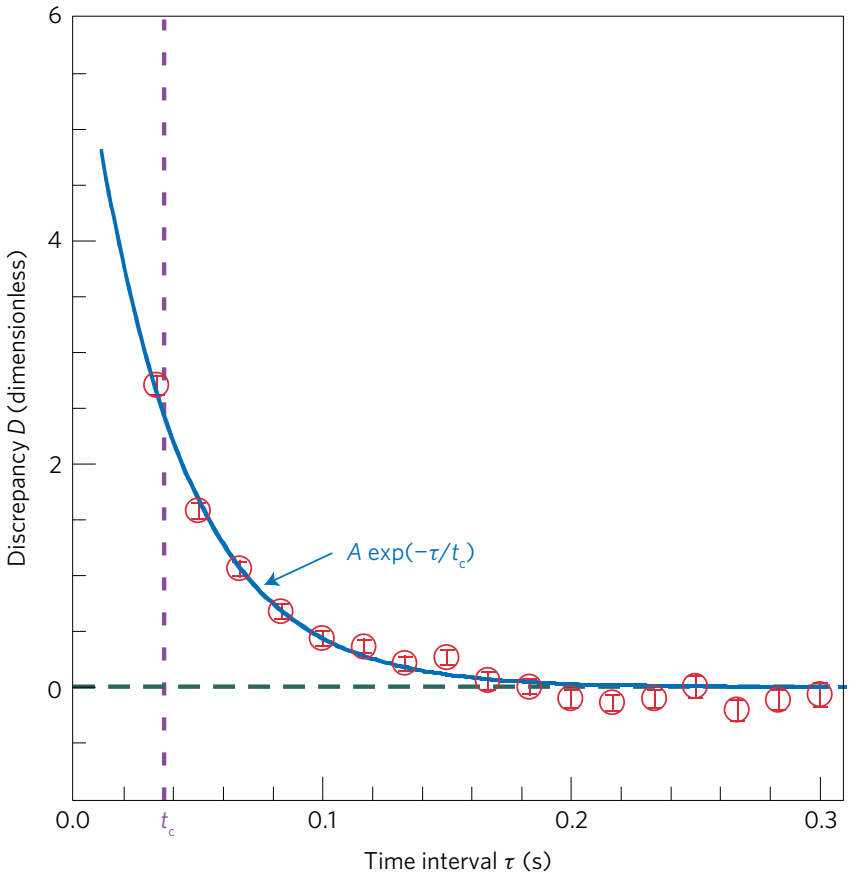

Figure 4 | Convergence of the ECM fluctuation theorem. Convergence as $\tau \rightarrow \infty$ is an essential aspect of the ECM fluctuation theorem. To quantify the timescale for this convergence, we begin by obtaining the discrepancy $D(\tau)$ between the slopes of two curves, LHS versus $C$ and RHS versus $C$, at a specified $\tau$. The uncertainty in computing the slopes for the LHS curves yielded the one-standard-deviation error bars of $D$. We find that convergence occurs with an exponential diminishment of $D$ for increasing $\tau$. A second fit of the data shown here to an exponential yields a convergence time $t_{\mathrm{c}}=0.037 \mathrm{~s}$.

where $D(\tau)$ is the difference in the slope of two curves: LHS versus $C$ and RHS versus $C$, at a specified value of $\tau$. For our experiment, the curve $D(\tau)$ decays exponentially, allowing us to define its e-folding time as $t_{c}$.

For our experiment, we find a convergence time $t_{\mathrm{c}}=0.037 \mathrm{~s}$ This value describes the time required for stochastic effects to begin accumulating in our shear flow. For our experimental conditions, $t_{\mathrm{c}}$ is comparable to the inverse of the mean entropy production rate of $0.053 \mathrm{~s}$ in Fig. 2, and $t_{\mathrm{c}}$ is also comparable to the inverse Einstein frequency (see Supplementary Information) $\Omega_{\mathrm{E}}^{-1}=0.024 \mathrm{~s}$.

Data availability. The data that support the plots within this paper and other findings of this study are available from the corresponding author upon reasonable request.

Received 30 December 2016; accepted 10 August 2017; published online 11 September 2017

\section{References}

1. Evans, D. J. \& Searles, D. J. The fluctuation theorem. Adv. Phys. 51, 1529-1585 (2002).

2. Ritort, F. Nonequilibrium fluctuations in small systems: from physics to biology. Adv. Chem. Phys. 137, 31-123 (2008).

3. Sevick, E. M., Prabhakar, R., Williams, S. R. \& Searles, D. J. Fluctuation theorems. Annu. Rev. Phys. Chem. 59, 603-633 (2008).

4. Jarzynski, C. Equalities and inequalities: irreversibility and the second law of thermodynamics at the nanoscale. Annu. Rev. Condens. Matter Phys. 2, 329-351 (2011).

5. Wang, G. M., Sevick, E. M., Mittag, E., Searles, D. J. \& Evans, D. J. Experimental demonstration of violations of the second law of thermodynamics for small systems and short time scales. Phys. Rev. Lett. 89, 050601 (2002).
6. Collin, D. et al. Verification of the Crooks fluctuation theorem and recovery of RNA folding free energies. Nature 437, 231-234 (2005).

7. Garnier, N. \& Ciliberto, S. Nonequilibrium fluctuations in a resistor. Phys. Rev. E 71, 060101 (2005).

8. Alemany, A., Mossa, A., Junier, I. \& Ritort, F. Experimental free-energy measurements of kinetic molecular states using fluctuation theorems. Nat. Phys. 8, 688-694 (2012).

9. Koski, J. V. et al. Distribution of entropy production in a single-electron box Nat. Phys. 9, 644-648 (2013).

10. Ciliberto, S., Joubaud, S. \& Petrosyan, A. Fluctuations in out-of-equilibrium systems: from theory to experiment. J. Stat. Mech. 2010, P12003 (2010).

11. Ichimaru, S. Strongly coupled plasmas: high-density classical plasmas and degenerate electron liquids. Rev. Mod. Phys. 54, 1017-1059 (1982).

12. Morfill, G. E. \& Ivlev, A. V. Complex plasmas: an interdisciplinary research field. Rev. Mod. Phys. 81, 1353-1404 (2009).

13. Hazeltine, R. D. \& Waelbroeck, F. L. The Framework of Plasma Physics Ch. 1 (Westview Press, 2004).

14. Evans, D. J., Cohen, E. G. D. \& Morriss, G. P. Probability of second law violations in shearing steady states. Phys. Rev. Lett. 71, 2401-2404 (1993).

15. Merlino, R. L. \& Goree, J. A. Dusty plasmas in the laboratory, industry, and space. Phys. Today 57, 32-38 (July, 2004).

16. Fortov, V. E., Ivlev, A. V., Khrapak, S. A., Khrapak, A. G. \& Morfill, G. E. Complex (dusty) plasmas: current status, open issues, perspectives. Phys. Rep. 421, 1-103 (2005).

17. Bonitz, M., Henning, C. \& Block, D. Complex plasmas: a laboratory for strong correlations. Rep. Prog. Phys. 73, 066501 (2010).

18. Feng, Y., Goree, J., Liu, B. \& Cohen, E. G. D. Green-Kubo relation for viscosity tested using experimental data for a two-dimensional dusty plasma. Phys. Rev. E 84, 046412 (2011).

19. Whipple, E. C. Potentials of surfaces in space. Rep. Prog. Phys. 44, 1197-1250 (1981).

20. Pramanik, J., Prasad, G., Sen, A. \& Kaw, P. K. Experimental observations of transverse shear waves in strongly coupled dusty plasmas. Phys. Rev. Lett. 88, 175001 (2002)

21. Killian, T. C. et al. Creation of an ultracold neutral plasma. Phys. Rev. Lett. 83, 4773-4779 (1999).

22. Koenig, M. et al. Progress in the study of warm dense matter. Plasma Phys. Control. Fusion 47, B441-B449 (2005).

23. Nosenko, V., Goree, J. \& Piel, A. Laser method of heating monolayer dusty plasmas. Phys. Plasmas 13, 032106 (2006).

24. Haralson, Z. \& Goree, J. Laser heating of 2-D dusty plasmas using a random arc pattern. IEEE Trans. Plasma Sci. 44, 549-552 (2016).

25. Io, C.-W. \& Lin, I. Steady-shear-enhanced microdiffusion with multiple time scales of confined, mesoscopic, two-dimensional dusty-plasma liquids. Phys. Rev. E 80, 036401 (2009).

26. Hartmann, P., Sándor, M. C., Kovács, A. \& Donkó, Z. Static and dynamic shear viscosity of a single-layer complex plasma. Phys. Rev. E 84, 016404 (2011)

27. Feng, Y., Goree, J. \& Liu, B. Observation of temperature peaks due to strong viscous heating in a dusty plasma flow. Phys. Rev. Lett. 109, 185002 (2012)

28. Gallavotti, G. \& Cohen, E. G. D. Dynamical ensembles in nonequilibrium statistical mechanics. Phys. Rev. Lett. 74, 2694-2697 (1995).

29. Sanbonmatsu, K. Y. \& Murillo, M. S. Shear viscosity of strongly coupled yukawa systems on finite length scales. Phys. Rev. Lett. 86, 1215-1218 (2001).

30. Feng, Y., Goree, J. \& Liu, B. Accurate particle position measurement from images. Rev. Sci. Instrum. 78, 053704 (2007).

\section{Acknowledgements}

We thank R. Belousov and E. G. D. Cohen for helpful discussions. This work was supported by the US National Science Foundation, the US Department of Energy, and NASA.

\section{Author contributions}

C.-S.W. and J.G. conceived and designed the experiment; Z.H. performed the experiment; C.-S.W. and J.G. analysed the data; B.L. performed the numerical simulations; C.-.S.W. and J.G. wrote the paper.

\section{Additional information}

Supplementary information is available in the online version of the paper. Reprints and permissions information is available online at www.nature.com/reprints. Publisher's note: Springer Nature remains neutral with regard to jurisdictional claims in published maps and institutional affiliations. Correspondence and requests for materials should be addressed to C.-S.W.

\section{Competing financial interests}

The authors declare no competing financial interests. 